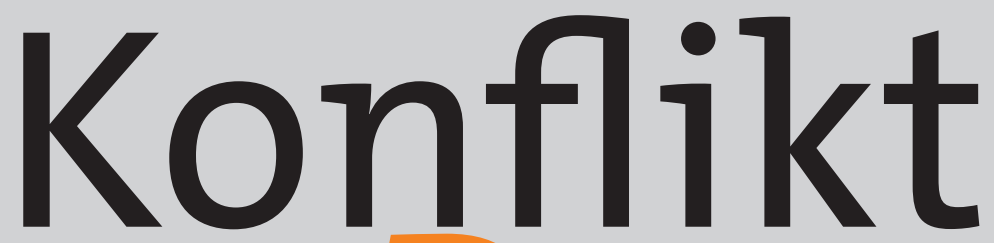

konfliktdynamik.nomos.de
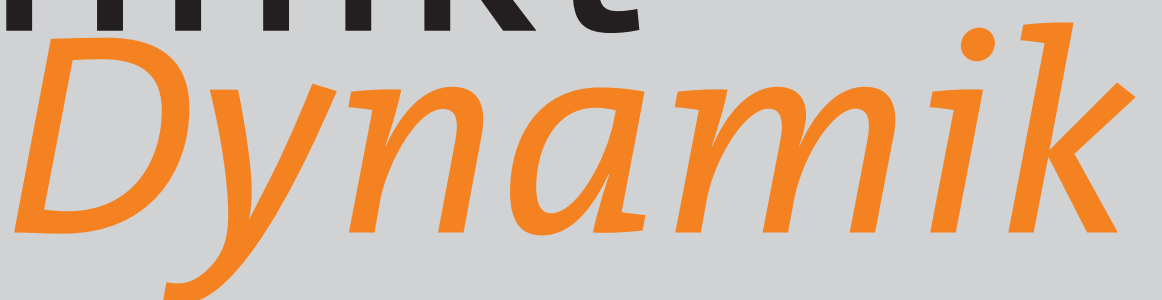

$2 \mid 2019$

8. Jahrgang 2019

ISSN 2193-0147

Verhandeln, Vermitteln und Entscheiden in Wirtschaft und Gesellschaft

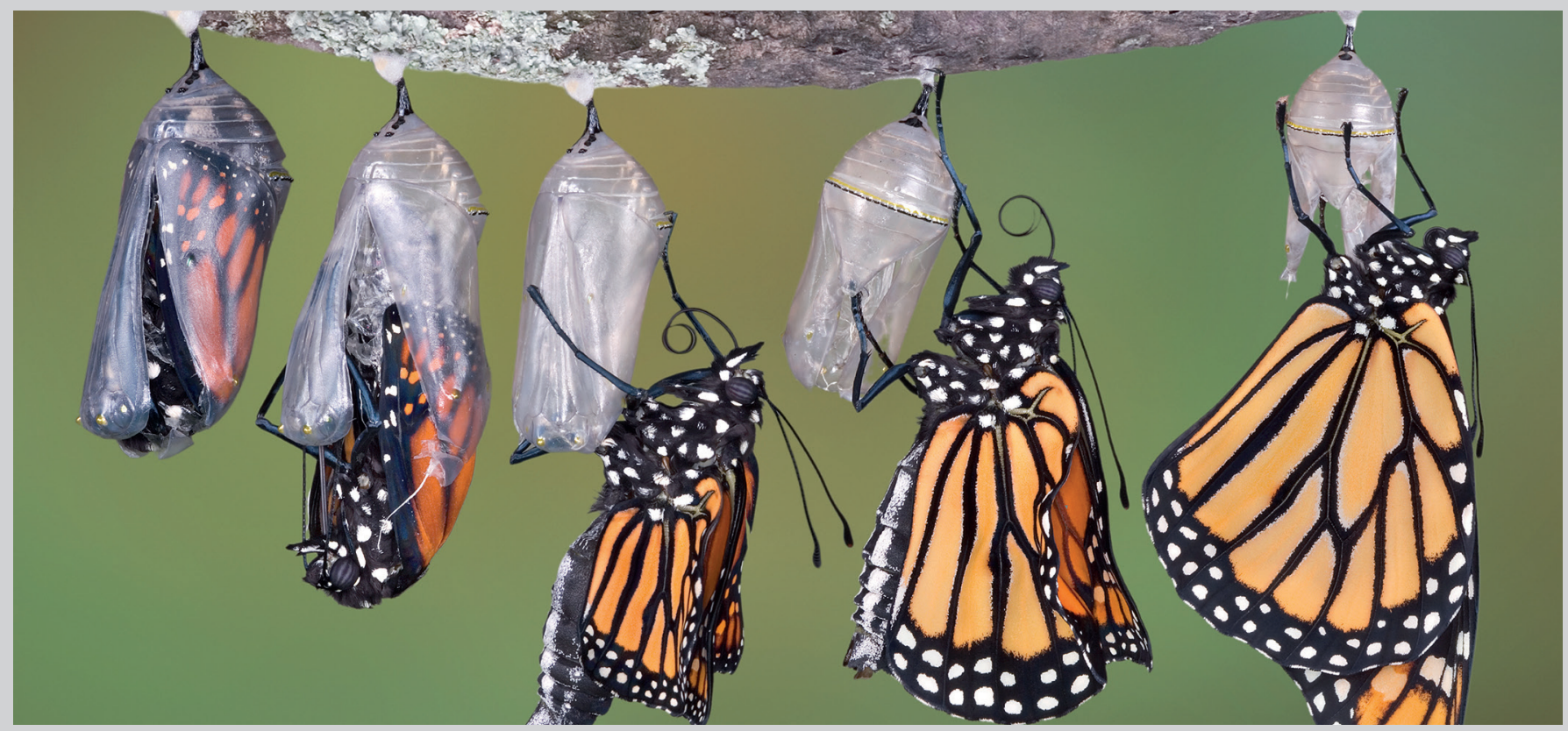

\title{
Kulturtransformationen in Organisationen
}

Herausgegeben von

Markus Troja

Alexander Redlich

Renate Dendorfer-Ditges

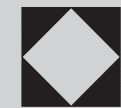

\section{Nomos}

Im Fokus

Wie ein nachhaltiger Musterwechsel

befeuert werden kann - ein

Erfahrungsbericht

Das harte Problem bei der Veränderung einer Organisationskultur und warum wir es nicht anschauen möchten

Wie Kulturtransformation in

Organisationen gestaltet werden kann

Theorie und Praxis

Partizipation garantiert keine Legitimation Musik und Konflikt

Konfliktklärung an Hochschulen

\section{Interview}

Kulturkonflikte in Organisationen als Hebamme der Innovation Rudi Ballreich im Gespräch mit Friedrich Glasl

\section{Methoden}

SK-Prinzip - Systemisches Konsensieren 2.0

- In Medias Res Juris

Steter Tropfen höhlt den Stein

Kolumne

Kreativ Leben? 
Liebe Leserin, lieber Leser,

Wer sich mit Konflikten in Organisationen beschäftigt, hört oft, dass sich der Druck in den letzten IO-I5 Jahren erhöht habe, vor allem durch Internationalisierung, Digitalisierung, Beschleunigung und Arbeitsverdichtung. Skandale und Fehlverhalten gehören zu den Folgen. Auch der Ton werde dadurch härter und Verhandlungen konfrontativer, sowohl intern als auch mit Kunden, Lieferanten und anderen Stakeholdern. Bei solch grundsätzlichen und strukturellen Konfliktfaktoren scheinen Schulungen und Tools auf der Verhaltensebene zu kurz zu greifen. Wenn die geforderten Werte und Verhaltensweisen eines wertschätzenden und kooperativen Umgangs im Widerspruch stehen zu der erlebten Kultur im Arbeitsalltag, führen die Appelle eher zu Zynismus. Es ist klar, dass die Veränderung tiefer greifen muss. Es geht um die Kultur der Organisation im Innen und Außen. Die Krisen mancher Großunternehmen haben daher zu Kulturveränderungsprogrammen geführt, allerdings mit sehr gemischtem Erfolg. Solche Kulturveränderungen in Organisationen stehen im FOKUS dieser Ausgabe. Was gehört dazu, woran scheitern sie, welche Ansätze und Erfahrungen gibt es? RUDI BALLREICH hat den Themenschwerpunkt betreut und gibt in seinem Einführungstext einen Überblick über die einzelnen Beiträge.

In der Rubrik THEORIE UND PRAXIS befasst sich MATTHIAS MEIFERT mit partizipativen Prozessen der Umgestaltung von Organisationen. Dazu greift er auf die Strukturationstheorie von Anthony Giddens zurück. Anhand eines konkreten Falls stellt er die Transformation von Strukturen und kulturellen Aspekten eines Unternehmensbereichs dar. »Organisation« wird dabei eher als soziale Praxis denn als Institution verstanden. Handlungen beruhen auf Strukturen, stabilisieren und verändern sie zugleich. Es wird deutlich, dass ein partizi- pativ angelegter Transformationsprozess mannigfaltige Konflikte zur Folge hat.

Der Konflikt zwischen dem künstlerischen Leiter des Orchesters der Berliner Staatsoper, Daniel Barenboim, und ehemaligen Musikern ging durch die Medien und führte zur Einrichtung einer Konfliktklärungsstelle für das Orchester. Bei den Auseinandersetzungen ging es auch um unterschiedliche Bewertungen des musikalischen Anspruchs. HANS-PETER ACHBERGER bietet in seinem Beitrag einen neuen Blick auf solche Konflikte in der professionellen Orchestermusik. Es gibt bereits Erfahrungsberichte über Konflikte und Mediation in Orchestern. Allerdings geht es dabei meist um zwischenmenschliche und organisatorische Konflikte, wie sie auch in anderen Teams auftreten können. Achberger beschreibt dagegen, wie die Musik selbst als Konfliktfaktor wirken kann. Zur Lösung solcher »musikalischer Konflikte« brauchen BeraterInnen und MediatorInnen ein musikalisches Verständnis, so Achberger. Er beschreibt Anforderungen an ein Konfliktmanagement in Orchestern.

Unter Rückgriff auf einschlägige Literatur fasst KLAUS MAAS in seinem Artikel zu Konflikten an Hochschulen die wichtigsten Besonderheiten der Organisation »Hochschule« zusammen, die zur Entstehung und Aufrechterhaltung von Konflikten beitragen können. Dabei kommt er zu drei Schwerpunkten (Autonomie des akademischen Bereichs, interne Widersprüche in der Promotionsausbildung und Teamarbeit), zu denen er praktische Hinweise für die Konfliktarbeit entwickelt.

FRIEDRICH GLASL spricht im INTERVIEW ebenfalls über Kulturveränderungen in Organisationen und erklärt die Probleme und Möglichkeiten anhand des Modells der Entwicklungsphasen von Organisationen, wie sie in den I970er Jahren von Bernhard Lievegoed beschrieben und von Glasl und Lievegoed weiterentwickelt wurden. Fried- rich Glasl erklärt insbesondere die Kulturkonflikte in den Phasenübergängen.

DOMINIK BERGER stellt in der METHODENRUBRIK anhand eines Falles die Anwendung des Systemischen Konsensieres im Einzelnen dar. Es handelt sich dabei um eine Entscheidungsmethode, die auf dem Grundgedanken beruht, die am wenigsten abgelehnte Option statt der von den meisten bevorzugten Alternative zu bestimmen. Der Autor beschreibt die Vorteile der Methode und skizziert, wie man dabei vorgehen kann.

RENATE DENDORFER DITGES und PHILIPP WILHELM informieren in der Rubrik IN MEDIAS RES IURIS über die Initiativen in Niedersachsen zur finanziellen Förderung der Mediation, die zumindest ein deutliches Signal sind, während der Gesetzgeber auf Bundesebene insoweit keine Initiative zeigt. Die Verankerung der Mediation im Rechtssystem führt derzeit insgesamt zu geringer Aufmerksamkeit bei der Bundesregierung.

In der KOLUMNE befasst sich BERND SCHMID mit dem Ideal der Kreativität im Hinblick auf unternehmerischen Erfolg. Ob Kreativität ausreicht, was noch dazugehört und welche Rolle Perspektivwechsel und Angst spielen - darüber kann man mit ihm nachdenken.

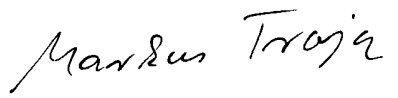

Markus Troja

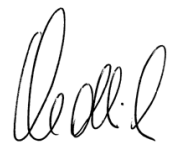

Alexander Redlich

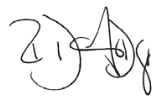

Renate Dendorfer-Ditges 


\section{Konflikt Dynamik $^{2}$ \\ Call for Papers}

\section{für die Zeitschrift Konfliktdynamik -}

\section{Verhandeln, Vermitteln und Entscheiden in Wirtschaft und Gesellschaft}

Die Konfliktdynamik schlägt eine Brücke zwischen Wissenschaft und Praxis konstruktiver Konfliktbehandlung. Zielgruppen sind Führungskräfte, Mediatoren, Coaches, Trainer, Organisationsberater sowie Wissenschaftler aller Disziplinen, die sich mit dem Thema Konflikt befassen. Die Zeitschrift veröffentlicht theoretisch, empirisch und praktisch fundierte Beiträge. Mittlerweile erscheint der vierte Jahrgang und die Rückmeldungen aus der Fachwelt zur Qualität der Artikel sind außerordentlich positiv.

Die Konfliktdynamik bietet den Leserinnen und Lesern Informationen in den Rubriken Theorie und Praxis, Impulse aus der Forschung, Aus den Organisationen, Hinter den Kulissen und Methoden. Überdies liefert sie in der Rubrik In medias res juris prägnante Informationen zu aktuellen juristischen Fragen des Konfliktmanagements, in einer Kolumne Nachdenkliches und unter Rezensionen Besprechungen wichtiger Literatur, Filme und Hörspiele.

Im Fokus jeder Ausgabe steht ein inhaltlicher Schwerpunkt mit mehreren Beiträgen zu unterschiedlichen Themen des Konfliktmanagements in Organisationen. Stichworte zu den Schwerpunktthemen in den nächsten Ausgaben lauten:

\section{Ausgabe 1/ 2020: Herausforderungen von organisationalen Transformationen meistern}

Ausgabe 2/ 2020: Mediationskritik

Ausgabe 3/2020: Weisheit im Führungsalltag

Ausgabe 4/ 2020: Wettkampf

Die Herausgeber bitten um Beiträge zu den genannten Rubriken und zu den Schwerpunktthemen. Es wird empfohlen, sich bereits während der Konzeption eines Beitrages mit der Redaktion abzustimmen: redaktion@konfliktdynamik.de. Für Fragen zu den Inhalten oder dem Publikationsverfahren stehen auch die Herausgeber gern zur Verfügung.

Die Konfliktdynamik erscheint vierteljährlich - jeweils Anfang Januar, April, Juli und Oktober.

Annahmeschluss für Beiträge ist jeweils 4 Monate vor dem Erscheinen. Alle Manuskripte durchlaufen ein Peer-Review-Verfahren.

Ausführliche Autorenhinweise finden sich unter www.konfliktdynamik.de. 


\section{Im Fokus}

Rudi Ballreich

Kulturtransformation in Organisationen . . . 92

\section{Annette Gebauer}

Kulturtransformation gestalten

Wie ein nachhaltiger Musterwechsel befeuert werden kann. Ein Erfahrungsbericht. . . . . . 994

\section{Werner Vogd}

Das harte Problem bei der Veränderung einer Organisationskultur und warum wir es nicht anschauen möchten . . . . . . . 104

\section{Rudi Ballreich}

Wie Kulturtransformation in Organisationen gestaltet werden kann (Teil 1)

Anregungen für Führungskräfte, Change

Agents und Beraterlnnen . . . . . . . . 112

\section{Theorie und Praxis}

\section{Matthias Meifert}

Partizipation garantiert keine Legitimation

Über die Konfliktträchtigkeit eines partizipativen

Transformationsprozesses im Human Ressource

Management einer Genossenschaftsbank . . 122

\section{Hans-Peter Achberger}

Musik und Konflikt

Gemeinsames Musizieren als möglicher

Konfliktherd .............. 132

\section{Klaus Maas}

Konfliktklärung an Hochschulen ...... 140

\section{Interview}

Kulturkonflikte in Organisationen als Hebamme der Innovation . . . . . . . . . 148

Rudi Ballreich im Gespräch mit Friedrich Glasl

\section{Methoden}

\section{Dominik Berger}

SK-Prinzip - Systemisches Konsensieren 2.0 . .

\section{Rezensionen}

Holger Lindemann, Claude-Hélène Mayer Q

Ilse Osterfeld (2018)

Systemisch-lösungsorientierte Mediation und

Konfliktklärung. . . . . . . . . . . . . . . 158

Meredith Haaf (2018)

Streit. Eine Aufforderung. . . . . . . . . . . . . . 159

Foad Forghani (2018)

Negotius - Dimensionen der

Verhandlungsführung . . . . . . . . . . . 160

Wilfried Kerntke (2018)

Wie Ziegen und Fische fliegen lernen. Die Entwicklungskraft von Konflikten in Unternehmen. . . . . 160

\section{In Medias Res Juris}

Renate Dendorfer-Ditges und Philipp Wilhelm

Steter Tropfen höhlt den Stein

Neuigkeiten zu Mediation aus den Ministerien • 162

\section{Kolumne}

\section{Bernd Schmid}

Kreativ Leben? . . . . . . . . . . . . 166

Kongresskalender . . . . . . . . . . 167

Vorschau ................ 168

Impressum . . . . . . . . . . 168 